\title{
Synthesizing Certified Code
}

\author{
Michael Whalen ${ }^{\dagger}$, Johann Schumann ${ }^{\ddagger}$, and Bernd Fischer ${ }^{\ddagger}$ \\ ${ }^{\dagger}$ Department of Computer Science and Engineering \\ Univ. of Minnesota, Minneapolis, MN 55455 \\ whalen@cs.umn.edu \\ ${ }^{\ddagger}$ RIACS / NASA Ames, Moffett Field, CA 94035 \\ \{schumann|fisch\}@email.arc.nasa.gov
}

\begin{abstract}
Code certification is a lightweight approach for formally demonstrating software quality. Its basic idea is to require code producers to provide formal proofs that their code satisfies certain quality properties. These proofs serve as certificates that can be checked independently. Since code certification uses the same underlying technology as program verification, it requires detailed annotations (e.g., loop invariants) to make the proofs possible. However, manually adding annotations to the code is time-consuming and error-prone.

We address this problem by combining code certification with automatic program synthesis. Given a high-level specification, our approach simultaneously generates code and all annotations required to certify the generated code. We describe a certification extension of AutoBayes, a synthesis tool for automatically generating data analysis programs. Based on built-in domain knowledge, proof annotations are added and used to generate proof obligations that are discharged by the automated theorem prover E-SETHEO. We demonstrate our approach by certifying operator- and memory-safety on a data-classification program. For this program, our approach was faster and more precise than PolySpace, a commercial static analysis tool.
\end{abstract}

Keyword: automatic program synthesis, program verification, code certification, proof-carrying code, automated theorem proving.

\section{Introduction}

Code certification is a lightweight approach to formally demonstrate software quality. It concentrates on aspects of software quality that can be defined and formalized via properties, e.g., operator safety or memory safety. Its basic idea is to require code producers to provide formal proofs that their code satisfies these quality properties. The proofs serve as certificates which can be checked independently, either by the code consumer or by certification authorities.

Code certification is an alternative to other, more established validation and verification techniques. It is more formal than code inspection and can show stronger properties than static analysis. In contrast to testing, code certification demonstrates that the properties of interest hold for all possible execution paths of the program. It also complements software model checking, which works on a 
different set of properties (typically liveness properties such as absence of deadlocks). Moreover, model checking does not produce explicit certificates: while it can produce counter-examples if a property is violated, validity follows only indirectly from the claim of an exhaustive search through the state space that cannot be checked independently.

In essence, code certification is a more tractable version of traditional axiomatic or Hoare-style program verification. It uses the same basic technology: the program is annotated with an axiomatic specification, the annotated program is fed into a verification condition generator (VCG) which produces a series of proof obligations, the proof obligations are proven or discharged by an automated theorem prover (ATP). The difference, however, is in the details: the certified properties are much simpler and much more regular than full behavioral specifications. Both aspects are crucial: Since the properties are much simpler, the resulting proof obligations are much simpler as well. Consequently, discharging them is also much easier; in many cases, all proof obligations can be shown fully automatically. Since the properties are much more regular, the annotations can be derived schematically from an explicitly formulated safety policy. Consequently, the specification effort - which can become overwhelming in traditional verification - is also much smaller.

However, code certification shares not only the underlying technology with Hoare-style program verification but also a fundamental limitation: in order to certify non-trivial programs or non-trivial properties, auxiliary annotations (e.g., loop invariants) are required. Since these annotations describe program-specific properties, the VCG cannot derive them automatically from the safety policy; instead, they must be provided manually by the software designer. This severely limits the practical usability of current certification approaches like proofcarrying code (PCC) [26].

In this paper we address this problem by combining code certification with automatic code generation from high-level specifications. Our idea is to use the high-level domain information "known" to the code generator to generate not only code but also all annotations required to certify that code. We believe that this idea is generally applicable to template-based code-generation systems through embedding additional properties into code templates. We demonstrate this embedding process for a particular program synthesis system, showing that the system formalizes enough high-level domain knowledge to generate all necessary annotations required to satisfy safety policies for complex programs. This domain knowledge cannot be recovered from the program by a certifying compiler as used in PCC. We further illustrate that state-of-the-art automated theorem provers can solve the verification conditions arising from the certification of such automatically synthesized annotated code. Moreover, we demonstrate that our approach can improve on conventional certification technology based on static program analysis.

This work represents an important step towards our long-term goal of extending a program synthesis system such that all generated programs can be certified completely automatically, thus relieving the users from having to anno- 
tate their code. This combination of program synthesis and program verification offers some unique benefits:

- It provides independent verification that automatically generated programs are safe.

- It can certify properties that are too "low-level" to be practically verified by the usual correct-by-construction arguments of the synthesis approaches.

- It can certify more complex properties and larger programs than certifying compilers.

- It can be tailored to provide proofs that function as audit trails for specific properties that are required for safety-critical software by regulatory agencies.

The remainder of the paper is organized as follows. In Section 2, we briefly describe methods and techniques underlying our approach: property verification, proof carrying code, and program synthesis. Section 3 contains a detailed architectural description of the certifying synthesizer. We specifically focus on how the safety-policy is reflected in extended Hoare-rules and how the annotations are produced and propagated during the synthesis process. We furthermore give a short description of the automated prover E-SETHEO and discuss results from processing the generated verification conditions. Section 4 covers related work and compares our approach to a conventional certification approach based on static analysis techniques. In Section 5 we conclude and sketch out future work.

\section{Background}

\subsection{Property Verification}

Traditionally, program verification has focused on showing the functional equivalence of (full) specification and implementation. However, this verification style is very demanding, because of the involved specification and proof efforts, respectively. Therefore, more recent approaches concentrate on showing specific properties that are nevertheless important for software safety. For example, model checking has been used successfully to verify liveness and safety aspects of distributed software systems [36]. We extend this property-oriented verification style in two key aspects. First, we use automated theorem provers for full first-order logic that do not require abstractions and that produce the necessary "real" proofs that can be checked independently. Second, we investigate how this approach can be extended towards a broader set of properties.

While many mechanisms and tools for verifying program properties have been published, especially for distributed systems, relatively little attention has been paid to the properties themselves. The related work in this area is usually concerned with computer security [32]; we are interested in all "useful" properties. To help guide our research, we have created an initial taxonomy of verifiable aspects of programs.

We first distinguish between functional and property-based verification. Functional verification is necessary to show that a program correctly implements a 
high-level specification. Typically, these proofs are performed by showing that a program is equivalent to, or a refinement of, some higher-level specification. Property-based verification, on the other hand, ensures that the programs have desirable features (e.g., absence of certain runtime errors), but does not show program correctness in the traditional sense. These properties are often much simpler to verify than full functional correctness; however, functional verification and property-based verification are not fundamentally different-in fact, many of the properties are necessary to show functional correctness.

These properties can be grouped into four categories: safety, resource-limit, liveness, and security properties. Safety properties prevent the program from performing illegal or nonsensical operations. Within this category, we further subdivide into five different aspects of safety:

Memory safety properties assert that all memory accesses involving arrays and pointers are within their assigned bounds.

Type safety properties assert that a program is "well typed" according to a type system defined for the language. This type system may correspond to the standard type system for the language, or may enforce additional obligations, such as ensuring that all variables representing physical quantities have correct and compatible units and dimensions [21].

Numeric safety properties assert that programs will perform arithmetic correctly. Potential errors include: (1) using partial operators with arguments outside their defined domain (e.g., division by zero), (2) performing computations that yield results larger or smaller than are representable on the computer (overflow/underflow), and (3) performing floating point operations which cause an unacceptable loss of precision.

Exception handling properties ensure that all exceptions that can be thrown within a program are handled within the program.

Environment compatibility properties ensure that a program is compatible with its target environment. Compatibility constraints specify hardware, operating systems, and libraries necessary for safe execution. Parameter conventions define constraints on program communication and invocation.

Resource limit properties check that the required resources (e.g., stack size) for a computation are within some bound. Liveness/progress properties are used to show that the program will eventually perform some required activity, or will not be permanently blocked waiting for resources. Security properties prevent a program from accidental or malicious tampering with the environment. Security policies regulate access to system resources, and are often enforced by authentication procedures, which determine the identity of the program or user involved.

Clearly, there is overlap between these categories; for example, many security flaws are due to safety violations. Our list also includes many properties that are difficult or impossible to automatically verify in the general case; we plan to extend and clarify this taxonomy in future work. For this paper, we have chosen 
to investigate two safety properties: array bounds checks and numeric partial operator/function domain errors.

\subsection{Proof-Carrying Code}

Proof-carrying code $[26,1]$ is a certification approach especially suited for mobile code. Many distributed systems (e.g., browsers, cellular phones) allow the user to download executable code and run it on the local machine. If, however, the origin of this code is unknown, or the source is not trustworthy, this poses a considerable risk: the dynamically loaded code may not be compatible with the current system status (e.g., operating system version, available resources), or the code can destroy (on purpose or not) critical data.

The concept of proof-carrying code has been developed to address the problem of showing certain properties (i.e., a safety policy) efficiently at the time when the software is downloaded. The developer of the software annotates the program which is subsequently compiled into object-code using a certifying compiler. Such a compiler (e.g., Touchstone [6]) carries over the source code annotations to the object-code level. A verification condition generator processes the annotated object code together with the public safety policy and produces a large number of proof obligations. If all of them are proven (by a theorem prover), the safety policy holds for this program. However, since these activities are performed by the producer, the provided proofs are not necessarily trustworthy. Therefore, the annotated code and a compressed copy of the proofs are packaged together and sent to the user. The user reconstructs the proof obligations and uses a proof checker to ensure that the conditions match up with the proofs as delivered with the software. Both the local VCG and the proof checker need to be trusted in this approach. However, since a proof checker is much simpler in its internal structure than a prover, it is simpler to design and implement it in a correct and trustworthy manner. Furthermore, checking a proof is very efficient, in stark contrast to finding the proof in the first place - which is usually a very complex and time-consuming process.

A number of PCC-approaches have been developed, particularly focusing on the compact and efficient representation of proofs (e.g., using LCF [26] or HOL [1]). However, as mentioned earlier, all of these approaches are in practice restricted to very simple properties. More intricate properties require the producer of the program to provide elaborate annotations and to carry out complicated formal proofs manually.

\subsection{Program Synthesis}

Automated program synthesis aims at automatically constructing executable programs from high-level specifications. Although a variety of approaches exist [19], we will focus in this paper on a specific system, AutoBayes [11]. AutoBAYES generates complex data analysis programs (currently up to 1200 lines of $\mathrm{C}++$ code) from compact specifications. Throughout this paper, we will 
use a simple but realistic classification example to introduce the application domain and to describe the main features of the underlying synthesis process.

Assume our task is to analyze spectral data measurements, e.g., from a star. Our instrument registers photons and their energy. All we know is that a photon originates from one of $M$ different sources which emit photons at different energy levels. The energy of each photon is not defined sharply but described by a normal distribution with a certain mean value and standard deviation. However, we do not know the mean and standard deviation for each source, nor do we know their relative strength (i.e., the percentage of photons coming from each individual source). Figure 1 shows an example data set for $M=3$ (see [4] for the physical background).

A statistical model can be written down easily and in a compact way. For the measurements $x_{0}, \ldots, x_{N-1}$ we know that each point is normal (Gaussian) distributed around the mean value $\mu$ with a standard deviation $\sigma$ for the class (individual source) $c_{i}$ to which the photon belongs, i.e., $x_{i} \sim N\left(\mu_{c_{i}}, \sigma_{c_{i}}^{2}\right)$ These class assignments $c_{i}$ and the relative class percentages $\phi$ are not known. All we know is that all photons belong to one of the classes, i.e., $\sum_{i=1}^{M} \phi_{i}=1$, and that summing up the class assignments results in the desired percentages. These four formulas comprise the core of the problem specification.

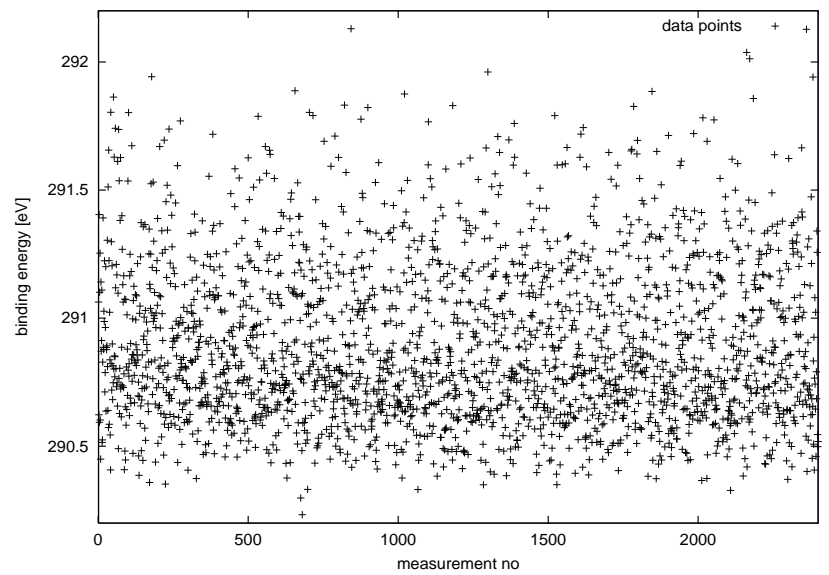

Fig. 1. Example spectral data for three sources $(M=3)$. The parameters are $\mu_{1}=$ 290.7, $\sigma_{1}=0.15, \phi_{1}=0.61, \mu_{2}=291.13, \sigma_{2}=0.18, \phi_{2}=0.33$, and $\mu_{3}=291.55, \sigma_{3}=$ $0.21, \phi_{3}=0.06$ (modeled after the spectrum of $N H_{3}$ molecules; cf. [4])

AutoBayes takes a specification similar to the formulas above (a total of 19 lines including all declarations, see [11] for details) and generates executable $\mathrm{C}++$ code of roughly 380 lines (including comments but not annotations) in less than a second on a $1000 \mathrm{MHz}$. SunBlade workstation. The implementation 
requires an iterative numerical algorithm ${ }^{1}$ which approximates the values of the desired variables $\mu, \sigma$, and $\phi$.

AutoBayes synthesizes code by exhaustive, layered application of schemas. A schema consists of a program fragment with open slots and a set of applicability conditions. The slots are filled in with code pieces by the synthesis system calling schemas in a recursive way. The conditions constrain how the slots can be filled; they must be proven to hold in the given specification before the schema can be applied. Some of the schemas contain calls to symbolic equation solvers, others contain entire skeletons of statistical or numerical algorithms. By recursively invoking schemas and composing the resulting code fragments, AutoBAYES is able to automatically synthesize programs of considerable size and internal complexity.

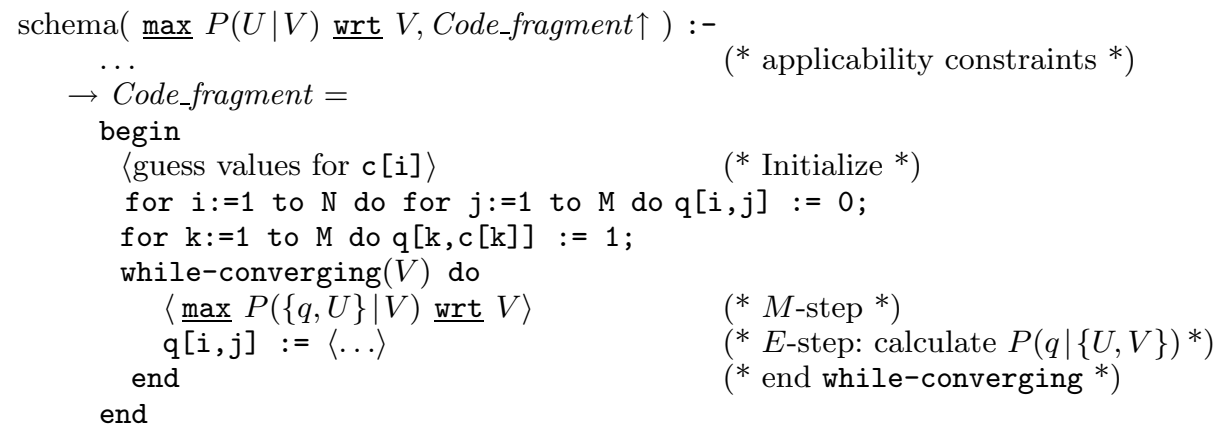

Fig. 2. EM-Schema (Fragment)

Let us consider the schema which is automatically selected as the core to solve our example. This schema, presented in a Prolog notation in Figure 2 above, solves the task to estimate the desired parameters ( $\phi, \mu, \sigma$ in our example) by maximizing their probability with respect to certain random variables (for a detailed description of the statistical background see [12]).

This task description is provided as a formal input parameter to the schema in Figure 2. The output parameter Code_fragment $\uparrow$ returns the synthesized code. After checking that the schema can be applied, the parts of the EM-algorithm are assembled. First, we generate a randomized initialization for the array q, then the iteration code starts. As in most numerical optimization algorithms (cf. $[15,29]$ ), we update the local array q until we have reached our desired accuracy (abbreviated as while-converging). The code fragments for the initialization of $\mathrm{q}$ and to calculate the updates (M-Step and E-Step) are constructed by recursively calling schemas on the respective subproblems. In Figure 2, these parts are included in $\langle\ldots\rangle$. Text set in typewriter font denotes code fragments in the target language; underlined words (like max) are keywords from AutoBAYES's specification language.

\footnotetext{
${ }^{1}$ Currently, an EM (expectation maximization) algorithm is generated.
} 
While we cannot present details of the synthesis process here, we want to emphasize that the code is assembled from building blocks which are obtained by symbolic computation or schema instantiation. The schemas clearly lay out the domain knowledge and important design decisions. As we will see later on, they can be extended in such a way that the annotations required for the certification are also generated automatically.

\subsection{Why Certify Synthesized Code?}

Program synthesis systems are usually built on the notion of "correctness-byconstruction." This means that the systems always produce code which correctly implement the user's specifications. Hence, the idea of explicitly certifying the synthesized code appears to be redundant. However, in practice, the notion of correctness-by-construction has two major problems which are mitigated by our approach.

First, the correctness-by-construction argument relies on only a single certificate - the synthesis proof. This proof is often extremely large and filled with artifacts from the domain theory. However, regulatory agencies overseeing the development of critical software systems describe very specific safety properties which need to be verified or tested [31]. Trying to extract the specific (sub-) proofs of these properties is difficult, if they are at all captured by the synthesis proof. Our approach allows a separation of proof concerns: any number of explicit and much smaller proofs can be generated, tailored towards the required safety properties.

Second, the validity of correctness-by-construction hinges on the correctness and consistency of the underlying synthesis engine and the domain theory. The synthesis engine is concerned with assembling schemas in a way that satisfies the constraints of the domain theory; thus, it ensures that the fragments of the program are correctly assembled. However, the code included in or constructed by the schemas for each fragment is not directly verified by the synthesis proof, so coding errors that are contained in the domain theory may be propagated through the synthesis process. Thus, users must in practice "trust" schemas to produce correct code, without independent verification of correctness. For safety/security sensitive code, e.g., for navigation/state estimation [39], this level of trust is not acceptable. Our approach provides an automatic and independent verification path for important properties of the generated code.

\section{System Architecture}

The architecture of our certifying synthesis system is somewhat similar to a typical proof-carrying code architecture. However, since we are currently not dealing with proof validation aspects, we only have three major building blocks (see Figure 3): the synthesis system AutoBAyes (which replaces the certifying compiler), the verification condition generator Mops, and the automated 
theorem prover E-SETHEO. All system components used in certification will be described in more detail below.

The system's input is a statistical model which defines the data analysis task as shown above. This specification need not be modified for certification - the process is thus completely transparent to the user. AutoBAYEs then attempts to synthesize code using the schemas described above. These schemas are extended appropriately (cf. Section 3.3) to support the automatic generation of code annotations. AutoBayes produces Modula- $2 \operatorname{code}^{2}$ which carries the annotations as formal comments. Annotations and code are then processed by the verification condition generator Mops. Its output is a set of proof obligations in first order predicate logic which must be proven to show the desired properties. In order to do so, a domain theory in form of an axiom set defining all operations and functions in the proof tasks must be added to the formulas. Finally, these extended proof obligations are fed into the automated theorem prover E-SETHEO.

For our prototype implementation, we added several small "glue" modules which convert the syntactical representation between all components. These are implemented in lex/yacc, awk, and Unix shell sh.

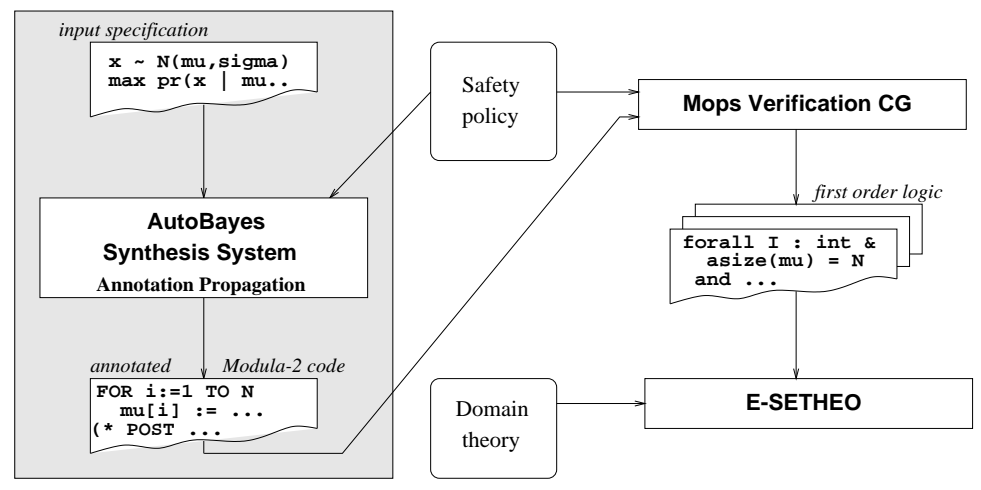

Fig. 3. AutoBayes system architecture, extended for code certification

\subsection{Safety Policy}

The first step in certification is to define precisely what constitutes safe behavior for the programs. In our case, we must define notions of memory and operator safety as predicates within a logic. Then a mechanism must be defined to transform a program into a series of verification conditions that are valid if and only if the safety properties are satisfied. In the sections below, we formulate the safety properties and describe the mechanism for creating the verification conditions.

${ }^{2}$ We extended AutoBayes to generate the Modula-2 code used by Mops. Usually, AutoBayes synthesizes C++/C programs for Octave [25] and Matlab [23]. 
Hoare rules [40] form the foundation of our approach. Hoare rules are triples of the form $\{P\} C\{Q\}$ where $C$ is a statement in an imperative programming language, and $P$ and $Q$ are predicates. The statement acts as a predicate transformer, that is, it describes how predicate $P$ is transformed into predicate $Q$ by the execution of $C$. Our idea, following Dijkstra's well-defined expression predicate Def [8], is to strengthen the preconditions of the Hoare rules by adding explicit memory- and operator-safety constraints. The rules for our policy are shown in Figure 4.

Array Bounds Safety Policy To show array bounds safety, we need to define variables within the assertion language that represent the notion of array bounds. Given these variables, we can test that each subscript expression within a statement is within the appropriate dimension for the array. More concretely, we assume that given an array $x$ of dimension $n+1$, all array dimensions $0 \leq k \leq n$ have the lower bound zero, and a size that is represented by $\operatorname{ASIZE}(x, k)$. The $A S I Z E(x, k)$ notation is used to denote the unique variable representing that array size.

We can then determine what it means for an expression to be array-bounds safe. Given an expression $E$, we say that it is array bounds safe if every array subscript expression for variable $x$ in dimension $k$ is between zero and $A S I Z E(x, k)-1$. To check, we define a function $\operatorname{ArrayRefs}(E)$ that returns a set of pairs of the form $\left(x,\left\langle e_{0}, e_{1}, \ldots, e_{n}\right\rangle\right)$. Each pair describes an array reference, where $x$ is the array variable and $\left\langle e_{0}, e_{1}, \ldots, e_{n}\right\rangle$ is the sequence of subscript expressions used to access the array. Then, a safe array reference $\operatorname{SafeRef}\left(x,\left\langle e_{0}, e_{1}, \ldots, e_{n}\right\rangle\right)$ is:

$$
\operatorname{SafeRef}\left(x,\left\langle e_{0}, e_{1}, \ldots, e_{n}\right\rangle\right) \equiv \forall a: 0 . . n \bullet 0 \leq e_{a}<\operatorname{ASIZE}(x, a)
$$

From this predicate, we can define expression safety w.r.t. the array bounds safety policy as follows:

$$
\operatorname{SafeExpr}_{A}(E) \equiv \forall(x, s e q) \in \operatorname{ArrayRefs}(E) \bullet \operatorname{SafeRef}(x, \text { seq })
$$

These two predicates state that, for each array reference, every subscript expression is within bounds.

Unfortunately, the SafeRef and $\mathrm{SafeExpr}_{A}$ predicates are higher-order, as they quantify over expressions in the program syntax. Since ArrayRefs $(E)$ yields a finite set, we can expand these quantified predicates over variables and expressions into a sequence of first-order predicates. For example, given the statement:

$$
q[k, c[k]]:=1 / v ;
$$

$\operatorname{SafeExp}_{A}(E)$ yields the following safety predicate, once expanded:

$$
\begin{aligned}
0 \leq k \wedge k<\operatorname{ASIZE}(q, 0) \wedge 0 \leq c[k] \wedge c[k]<A S I Z E(q, 1) \wedge \\
0 \leq k \wedge k<\operatorname{ASIZE}(c, 0)
\end{aligned}
$$


By checking that all array subscripts are within bounds for each array reference for each expression, we can determine array bounds safety for the entire program.

Operator Safety Policy To show operator safety, we only need to show that all divisors are different from zero. All other partial operators, such as square root, are implemented as standard library functions, and not as programming language language constructs. The domain constraints on these functions are enforced by the respective procedure pre- and post-conditions.

To check for zero divisors, a function divisors $(E)$ is defined. This function returns the set of subexpressions of an expression $E$ that are used as divisors. From this function, we can define expression safety with respect to the operator safety policy as follows:

$$
\operatorname{SafeExpr}_{O}(E) \equiv \forall e \in \operatorname{divisors}(E) \bullet e \neq 0
$$

So, given the statement (1), $\operatorname{SafeExpr}_{O}(E)$ yields the safety predicate $v \neq 0$, once expanded.

Extended Hoare rules Given definitions for operator and array bounds safety for expressions, we can extend the Hoare rules with respect to these policies. First, we define SafeExpr $(E)$ as:

$$
\operatorname{SafeExpr}(E) \equiv \operatorname{SafeExpr}_{A}(E) \wedge \operatorname{SafeExpr}_{O}(E)
$$

Then the Hoare rules can be formulated as shown in Figure 4.

The first rule applies to array declarations. As described earlier, we create a variable $\operatorname{ASIZE}(x, k)$ to refer to the size of array $x$ at dimension $k$. The declaration of an array is an assignment of values to these variables. The rule works by replacing instances of $A S I Z E(x, k)$ in the postcondition of a statement with the variable declaration expression. For example, given an array declaration

$\operatorname{var} c:$ array $[n c l a s s e s]$ of REAL

and a postcondition $\operatorname{ASIZE}(x, 0)=$ nclasses, this rule generates the precondition nclasses $=$ nclasses which is obviously true.

The second rule, assignment of scalars, is the same as the standard Hoare assignment rule, except that it has a strengthened precondition that checks that the assignment expression is safe w.r.t. our safety policies.

The third rule describes assignment of array cells. Unlike scalar assignment, array cell assignment cannot be handled by simple substitution, because of the possibility of aliasing of array cells. Instead, we think of the array as describing a mapping function from cells to values. An assignment to a cell is an update of the mapping function, written as $x\left\{\left(e_{0}, e_{1}, \ldots, e_{n}\right) \rightarrow e\right\}$. This approach is the standard extension of the Hoare calculus to handle arrays and is described fully in [22]. We strengthen the precondition of this rule to ensure that both the 
Array Declaration:

$\left\{\begin{array}{l}P\left[e_{0} / A S I Z E(x, 0), \ldots, e_{n} / A S I Z E(x, n)\right] \wedge \\ \quad \operatorname{SafeExpr}\left(e_{0}\right) \wedge \operatorname{SafeExpr}\left(e_{1}\right) \wedge \\ \vdots \\ \operatorname{SafeExpr}\left(e_{n}\right)\end{array}\right\} \operatorname{var} x: \operatorname{array}\left[e_{0}, \ldots, e_{n}\right]$ of $Y\{P\}$

Scalar Assignment: $\{P[e / x] \wedge \operatorname{SafeExpr}(e)\} x:=e\{P\}$

Array Assignment: $\left\{\begin{array}{l}P\left[x\left\{\left(e_{0}, \ldots, e_{n}\right) \rightarrow e\right\}\right] \wedge \\ \quad \operatorname{SafeExpr}(e) \wedge \\ \operatorname{SafeExpr}\left(x\left[e_{0}, \ldots, e_{n}\right]\right)\end{array}\right\} x\left[e_{0}, \ldots, e_{n}\right]:=e\{P\}$

Conditional Stmt: $\frac{\{P \wedge b \wedge \text { SafeExpr }(b)\} c\{Q\} \quad(P \wedge \neg b \wedge \text { SafeExpr }(b) \Longrightarrow Q)}{\{P \wedge \operatorname{SafeExpr}(b)\} \text { if } b \text { then } c\{Q\}}$

While Loop: $\quad \frac{\{P \wedge b \wedge \text { SafeExpr }(b)\} c\{P \wedge \text { SafeExpr }(b)\}}{\{P \wedge \operatorname{SafeExpr}(b)\} \text { while } b \text { do } c\{P \wedge \neg b \wedge \operatorname{SafeExpr}(b)\}}$

For Loop: $\quad\left\{\begin{aligned} P & \wedge e_{0} \leq x \leq e_{1} \\ & \wedge \operatorname{SafeExpr}\left(e_{0}\right) \\ & \wedge \operatorname{SafeExpr}\left(e_{1}\right)\end{aligned}\right\} C\left\{\begin{aligned} P[(x+1) / x] & \wedge \operatorname{SafeExpr}\left(e_{0}\right) \\ & \wedge \operatorname{SafeExpr}\left(e_{1}\right)\end{aligned}\right\}$

$\left\{\begin{array}{r}P\left[e_{0} / x\right] \wedge \operatorname{SafeExpr}\left(e_{0}\right) \\ e_{0} \leq e_{1} \wedge \operatorname{SafeExpr}\left(e_{1}\right)\end{array}\right\}$ for $x:=e_{0}$ to $e_{1}$ do $C\left\{\begin{array}{r}P\left[\left(e_{1}+1\right) / x\right] \\ \wedge \operatorname{SafeExpr}\left(e_{0}\right) \\ \wedge \operatorname{SafeExpr}\left(e_{1}\right)\end{array}\right\}$

Sequence: $\quad \frac{\{P\} s_{0}\{R\} \quad\{R\} s_{1}\{Q\}}{\{P\} s_{0} ; s_{1}\{Q\}}$

Rule of Conseq: $\frac{P^{\prime} \Longrightarrow P \quad\{P\} C\{Q\} \quad Q \Longrightarrow Q^{\prime}}{\left\{P^{\prime}\right\} C\left\{Q^{\prime}\right\}}$

Fig. 4. Hoare rules with safety policy extensions

subscript expressions in the left-hand side and the assignment expression are safe.

The next three rules describe conditional and loop statements. They are the same as the standard Hoare rules, with strengthened preconditions to show that their expressions are safe. Finally, we define the standard Hoare rule of consequence, which states that we can always legally strengthen the precondition or weaken the postcondition of a statement. Soundness of all rules is obvious. 


\subsection{The Verification Condition Generator}

In practical proof-carrying code approaches, the safety policy is hardcoded into the VCG component of the certifying compiler. In our approach all required annotations are generated so that any VCG can be used. For our experiments, we used the VCG of the Modula Proving System Mops [17]. Mops is a Hoarecalculus based verification system for a large subset of the programming language Modula-2 [41], including pointers, arrays, and other data structures. The verification of REAL-arithmetics is idealized and ignores possible round-off errors. Mops supports the verification of arbitrary program segments and not only procedures or modules. The verification segments can be nested to break large proofs into manageable pieces.

Mops uses a subset of VDM-SL [7] as its specification language; this is interpreted here only as syntactic sugar for classical first-order logic. All annotations are written as Modula-2 comments enclosed in $(*\{\ldots\} *)$. Pre- and postconditions start with the keywords pre and post, respectively, loop invariants with a loopinv, and additional assertions with an assert (cf. Figure 5.)

\subsection{Annotations and their Propagation}

Annotating the large programs created by AutoBAYES requires careful attention to detail and many annotations. There are potentially dozens of loops requiring an invariant, and nesting of loops and if-statements can make it difficult to determine what is necessary to completely annotate a statement. The schema-guided synthesis mechanism of AUTOBAYES makes it easy to produce annotations local to the current statement, as the generation of annotations is tightly coupled to the individual schema. For this reason, we split the task of creating the statement annotations into two parts: creating local annotations during the run of AutoBAYes, and propagating the annotations through the code.

Local Annotations The local annotations for a schema describe the changes in variables made by the schema, without needing to describe all of the global information that may later be necessary for proofs.

During synthesis (i.e., at the time when the schemas are instantiated), the annotations are produced locally for each statement. Each loop is annotated with a schematic invariant and schematic pre- and postconditions describing how it changes variables within the program. The specific form of the invariants and assertions depends on the safety policy supported by the synthesis system. For example, the precondition in lines 1-3 in Figure 5 is required to show memory safety, more specifically, the safety of the nested array access in line 26 . The fact that this precondition is actually required is part of our domain knowledge and thus encoded within the schema. Obviously, a modification or extension of the supported safety policy requires corresponding modifications or extensions of the schemas. 


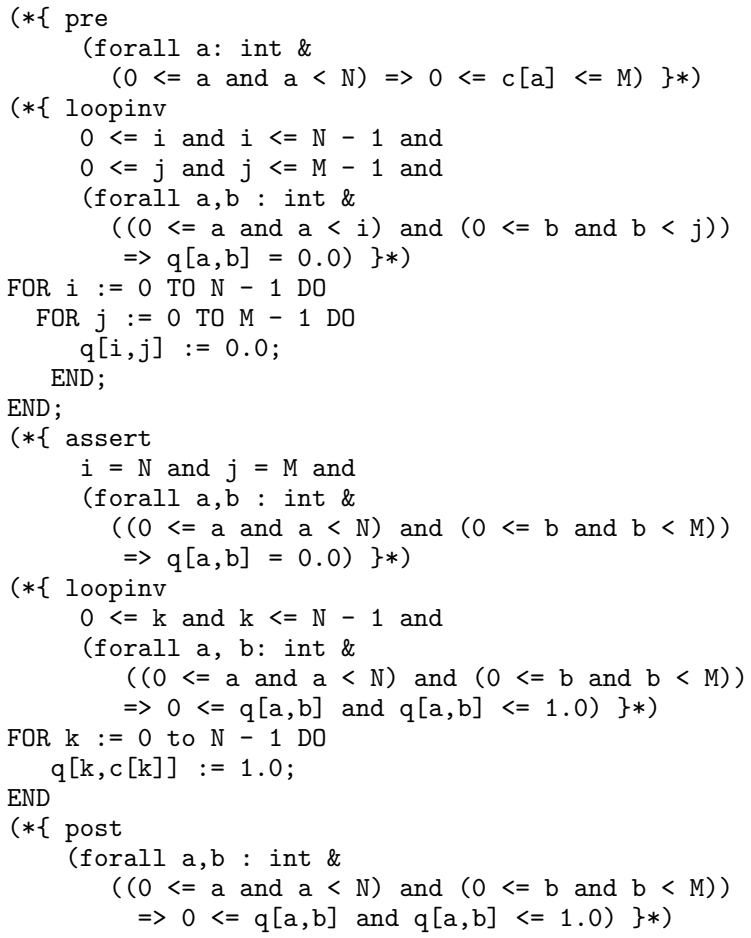

Fig. 5. Code fragment with annotations for the initialization of the intermediate arrays ( $\mathrm{q}$ and $\mathrm{c}$ ) as defined in the schema in Figure 2.

Propagation of Annotations Unfortunately, these local annotations are in general insufficient to prove the postcondition at the end of a larger code fragment. For example, at line 26 in the code (cf. Figure 5), we do not necessarily know what invariants hold prior to the loop. To overcome this problem, we propagate any unchanged information through the annotations. Because program synthesis restricts aliasing to few, known places, the test for which statements influence which annotations can be accomplished easily without full static analysis of the synthesized program.

In our example, we propagate the initial condition about the vector c (lines 1-3) and add it to the loop invariant and post-assertion for the first loop (lines 15-19). Since the second loop does not change variable c, this condition is propagated forward into invariant and post-condition of the second loop.

The propagation algorithm (shown in Figure 6) works on a tree. The tree nodes are initially labeled with the AutoBAYES-generated local annotations. The tree edges describe the locations of the annotations relative to one another in the code according to a lexicographic ordering which obeys the nesting of the language constructs. Each node may have many children in two categories: one sibling node and zero or more child nodes, corresponding to the lexical placement 
of the annotations in the code. The edges are labeled by the set of variables that have been assigned between the annotations.

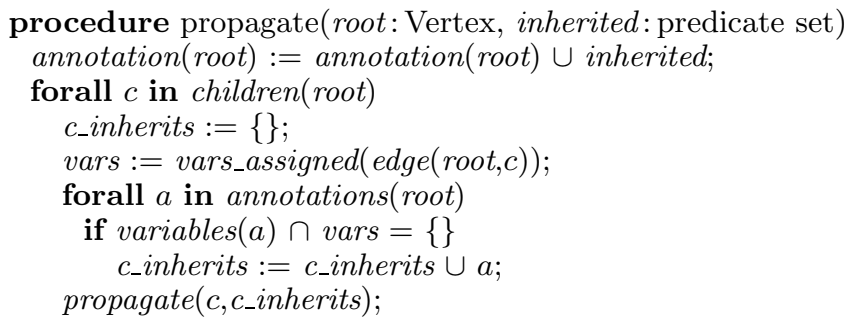

Fig. 6. The annotation propagation algorithm

The algorithm starts from the top of the tree and performs a recursive depthfirst traversal. The parameters to the algorithm are the current root node of the tree (root) and the set of inherited formulas (inherited). The algorithm first updates the annotations associated with the root node, annotation(root), to include the parent-node formulas. Then, for each child, it creates a set of inherited predicates (c_inherits) and calls itself recursively. The set $c \_i n h e r i t s$ is a set of all predicates from annotation(root) that do not contain variables modified by the intervening code. This information is extracted from the labeling of the edge between the root node and node $c$.

\subsection{The Automated Prover}

For our experiments we used the automated theorem prover E-SETHEO, version csp01 [5]. E-Setheo is a compositional theorem prover for formulas in first order logic, combining the systems E [33] and SETHEO [20,24]. The subsystems are based on the superposition, model elimination, and semantic tree calculi. Depending on syntactic characteristics of the input formula, an optimal schedule for each of the different strategies is selected. These different schedules have been computed from experimental data using machine learning techniques [35]. Because all of the subsystems work on formulas in clausal normal form (CNF), the first order formula is first converted into CNF using the module Flotter [38]. E-SETHEO is one of the most powerful ATP systems available as has been shown in recent international theorem proving competitions [5].

Out of the 69 proof tasks of our example, E-SETHEO initially could solve 65 automatically with a run-time limit of 120 seconds on a 1000 Mhz. SunBlade workstation. The remaining four proof tasks required some relatively simple preprocessing (splitting up the formula into two separate proof tasks) before they could be proven automatically. Most of the tasks could be solved in about one second, but several tasks took up to 20 seconds. The overall proof time of 323 seconds indicates that our approach is feasible. 


\section{Related Work}

We are not aware of any other work to automatically extract knowledge about the program under construction from the synthesis process, whether for certification or for other purposes. However, there is a large number of different approaches which share either techniques or goals with our work.

The approach most closely related to ours is proof-carrying code which has already been discussed in Section 2.2. However, due to its focus on mobile code, PCC covers many aspects we are (currently) not interested in, e.g., efficient proof representation and proof checking. It also works on the level of object code or typed intermediate languages (e.g., Flint [34]) and is thus complementary to our approach. Certifying compilers as Touchstone [6] or Cyclone [16] could consequently be used to show that the safety policy established on the source code level is not compromised by the compilation step.

Lowry et al. [21] present an approach for certifying domain-specific properties which is based on abstract interpretation. They check programs for frame safety, an extended type safety property. Other safety properties can also be encoded in extended type systems and then checked via (extended) type inference algorithms. Such approaches have been used to show, for example, unit and dimensional safety [30,18] and memory safety [42]. However, these approaches usually also require additional annotations, e.g., type declarations. Moreover, most of them are restricted to a specific safety policy and thus less general than proof-based certification approaches.

Many reverse engineering approaches try to recover formal specifications from code. Gannod and Cheng [14] use a strongest postcondition predicate transformer to support different reverse engineering tasks but their approach still requires additional manual annotations (e.g., loop invariants). Ernst et al. [9] try to infer such invariants dynamically, using a generate-and-test approach: potential invariants are generated from a set of patterns and checked against previously collected run-time trace information. However, the inferred predicates are not proven to be actually invariant so that the approach is not suitable for certification purposes. Flanagan and Leino [13] describe a similar system, Houdini, to support their ESC/Java verification system. Houdini also uses a generate-andtest approach but the test phase relies on ESC/Java to prove the invariants. However, Houdini does not use domain knowledge in the generate phase and is thus restricted in the kind of invariants it can recover.

Obviously, our research is also related to standard program verification. However, program verification concentrates on showing full functional equivalence or refinement between specifications and programs. This is true especially for integrated development/proof environments as for example the KIV system [37], and SPARK Ada [2]. Unlike our approach, program verification systems usually offer no support to find and formalize the functional specifications and auxiliary annotations. Our work could be used as a front-end, providing the necessary annotations for safety properties to these kinds of systems.

It is sometimes possible to encode aspects of the safety policy into the logic used in a program verification system. For example, if VDM is not interpreted 
as mere syntactic sugar for classical logic but as notation for the three-valued logic of partial functions (LPF) [3], a partial correctness proof in Mops already gives operator safety. Partial operators provide a uniform notion applicable for several safety properties, but they also preclude our ability to create small, separate proofs for different partial operator properties and require additional tool support.

Certification tools based on static analysis techniques try to show which parts of the code are safe with respect to a (usually hard-wired) safety policy. All necessary information is extracted from the code; hence, no annotations or domain knowledge are required. In order to compare our approach to static analysis, we analyzed the equivalent C-version of our example program with the tool PolySpace [28]. PolySpace was capable of declaring most of the code safe with respect to memory/operator safety. However, it could not clear several important parts of the code, most notably the nested indexing ( $[\mathrm{k}, \mathrm{c}[\mathrm{k}]]$, see line 26 in Figure 5) and the initialization of some variables in the main loop. In these cases, certification requires annotation propagation as it is done in our work; PolySpace does not require or support annotations. On the other hand, PolySpace detected a possible integer overflow error of a loop counter in the synthesized code, something that our safety policy does not (yet) check. The runtime of PolySpace for this example (about one hour of wall-clock time on the same machine as used for our experiments) demonstrates that our approach can be competitive to commercial tools.

\section{Conclusions}

In this paper, we have described a novel combination of automated program synthesis and automated program verification. Our basic idea is to generate the program together with detailed formal annotations which are required for a fully automatic correctness proof. This approach is facilitated by the knowledge of the domain and the program under construction which are formalized in the program synthesis system. Since it is virtually impossible to re-generate this information from the synthesized program only, our approach is much more powerful and "smarter" than a certifying compiler and allows us to certify complex properties for mid-sized programs fully automatically.

We have demonstrated the feasibility of our approach by certifying operator safety and memory safety for an automatically generated iterative data classification program. The synthesized program consists of roughly 380 lines of code, 90 of which are auto-generated comments to explain the code. With all annotations (including propagated annotations), it grows to 2,116 lines of code - a clear indication than manual annotation is out of question. The annotated program induces 69 proof tasks in first-order logic. After some minor preprocessing steps, all these tasks can be solved automatically in relatively short time, using the theorem prover E-SETHEO. In contrast, certification of this program using the commercial tool PolySpace took approximately one hour and was incomplete with respect to our chosen safety policy. 
Our long-term goal is to extend AutoBAyes such that all generated programs can be certified completely automatically. We are confident that our approach can also be extended to other program synthesis systems, because they generally encode enough abstract knowledge about the domain and the program under construction. We see a number of benefits from this combination of program synthesis and program verification. For the user of such a certifying synthesis system, the major benefit is obviously the additional verification of (important aspects of) the synthesized code; moreover, it comes at no cost for the user, and it can be double-checked independently.

This independent verification complements the notion of "correctness-byconstruction" generally built into program synthesis systems. This notion means that the system always produces code which correctly implements the user's specification. However, its validity depends on the correctness and consistency of the underlying synthesis engine and the domain theory. Because these are large and complex artifacts - comparable to a compiler - current technology cannot guarantee their correctness. Thus, a user must in reality "trust" that the synthesis system produces correct code. Our approach provides a tool and methodology to demonstrate important properties of the code in an automatic and independently re-checkable way.

Our system is still a prototype; the certification extension covers only those parts of the domain theory required to generate EM-variants. However, we see no fundamental obstacles in extending the approach to the entire (still growing) domain theory. Also, the safety-policy is hard-coded in the way the annotations are generated within the synthesis schemas. We will work on ways to explicitly represent safety policies (e.g., using higher-order formulations) and use this to tailor the annotation generation in AUTOBAYES. Our propagation algorithm can be viewed as a mechanism for managing proof context information. We are investigating more complex proof frameworks, such as program window inference [27] that provide rules for managing this information, making the propagation step of our approach unnecessary. Our architecture also relies on the correctness of E-SeTHEO. We are planning to extend our system to incorporate a small and verified proof checker which is able to give us the certainty that the proofs produced by E-SETHEO are indeed correct. Furthermore, we plan to implement a small and trustworthy verification condition generator.

Acknowledgements. We would like to thank the reviewers for their detailed and helpful comments. This work is supported by the NASA, grant 749-1011 (Thinking Systems / Program Synthesis); M. Whalen was supported by the RIACS SSRP program.

\section{References}

1. A. W. Appel and A. P. Felty. A semantic model of types and machine instructions for proof-carrying code. In Proc. 27th ACM Symp. Principles of Programming Languages, pp. 243-253. ACM Press, 2001.

2. J. Barnes. High Integrity Ada: The SPARK Approach. Addison-Wesley, 1997. 
3. H. Barringer, J. H. Cheng, and C. B. Jones. A logic covering undefinedness in program proofs. Acta Informatica, 21(3):251-269, Oct. 1984.

4. J. Berkowitz. Photoabsorption, Photoionization, and Photoelectron Spectroscopy. Academic Press, 1979.

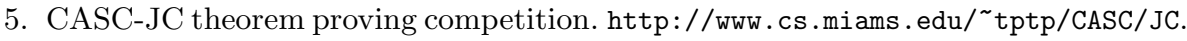

6. C. Colby, P. Lee, G. C. Necula, F. Blau, M. Plesko, and K. Cline. A certifying compiler for Java. ACM SIGPLAN Notices, 35(5):95-107, 2000.

7. J. Dawes. The VDM-SL Reference Guide. Pitman, London, 1991.

8. E. W. Dijkstra. A Discipline of Programming. Prentice-Hall, 1976.

9. M. D. Ernst, J. Cockrell, W. G. Griswold, and D. Notkin. Dynamically discovering likely program invariants to support program evolution. IEEE Trans. Software Engineering, 27(2):1-25, Feb. 2001.

10. M. S. Feather and M. Goedicke (eds.) Proc. 16th Intl. Conf. Automated Software Engineering, IEEE Comp. Soc. Press, 2001.

11. B. Fischer and J. Schumann. AutoBayes: A system for generating data analysis programs from statistical models. J. Functional Programming, 2002. To appear. Preprint available at http://ase.arc.nasa.gov/people/fischer/.

12. B. Fischer, J. Schumann, and T. Pressburger. Generating data analysis programs from statistical models (Position Paper). In W. Taha (ed.), Proc. Intl. Workshop Semantics, Applications, and Implementation of Program Generation, Lect. Notes Comp. Sci. 1924, pp. 212-229. Springer, 2000.

13. C. Flanagan and K. R. M. Leino. Houdini, an annotation assistant for ESC/Java. In J. Oliveira and P. Zave (eds.), Proc. Intl. Symp. Formal Methods Europe 2001: Formal Methods for Increasing Software Productivity, Lect. Notes Comp. Sci. 2021, pp. 500-517. Springer, 1997.

14. G. C. Gannod, Y. Chen, and B. H. C. Cheng. An automated approach for supporting software reuse via reverse engineering. In D. F. Redmiles and B. Nuseibeh (eds.), Proc. 13th Intl. Conf. Automated Software Engineering, pp. 79-86. IEEE Comp. Soc. Press, 1998.

15. P. Gill, W. Murray, and M. Wright. Practical Optimization. Academic Press, 1981.

16. L. Hornof and T. Jim. Certifying compilation and run-time code generation. Higher-Order and Symbolic Computation, 12(4):337-375, 1999.

17. T. Kaiser, B. Fischer, and W. Struckmann. Mops: Verifying Modula-2 programs specified in VDM-SL. In Proc. 4th Workshop Tools for System Design and Verification, pp. 163-167. 2000.

18. A. Kennedy. Programming Languages and Dimensions. $\mathrm{PhD}$ thesis, University of Cambridge, Apr. 1996. Published as UCCL TR391.

19. C. Kreitz. Program synthesis. In W. Bibel and P. H. Schmitt (eds.), Automated Deduction - A Basis for Applications, Vol III, pp. 105-134. Kluwer, 1998.

20. R. Letz, J. Schumann, S. Bayerl, and W. Bibel. SETHEO: A high-performance theorem prover. J. Automated Reasoning, 8(2):183-212, 1992.

21. M. Lowry, T. Pressburger, and G. Rosu. Certifying domain-specific policies. In Feather and Goedicke [10], pp. 118-125.

22. D. C. Luckham and N. Suzuki. Verification of array, record, and pointer operations in Pascal. ACM Trans. Programming Languages and Systems, 1(2):226-244, 1979.

23. C. B. Moler, J. N. Little, and S. Bangert. PC-Matlab Users Guide. Cochituate Place, 24 Prime Park Way, Natick, MA, USA, 1987.

24. M. Moser, O. Ibens, R. Letz, J. Steinbach, C. Goller, J. Schumann, and K. Mayr. The model elimination provers SETHEO and E-SETHEO. J. Automated Reasoning, 18:237-246, 1997. 
25. M. Murphy. Octave: A free, high-level language for mathematics. Linux Journal, 39, July 1997.

26. G. C. Necula and P. Lee. Efficient representation and validation of logical proofs. In Proc. 13th Annual IEEE Symp. Logic in Computer Science, pp. 93-104. IEEE Comp. Soc. Press, 1998.

27. R. Nickson and I. J. Hayes. Supporting contexts in program refinement. Science of Computer Programming, 29(3):279-302, 1997.

28. PolySpace technologies. http://www.polyspace.com, 2002.

29. W. H. Press, B. P. Flannery, S. A. Teukolsky, and W. T. Vetterling. Numerical Recipes in C. Cambridge Univ. Press, Cambridge, UK, 2nd. edition, 1992.

30. M. Rittri. Dimension inference under polymorphic recursion. In Proc. 7th Conf. Functional Programming Languages and Computer Architecture, pp. 147159, ACM Press, 1995.

31. Software Considerations in Airborne Systems and Equipment Certification. Radio Technical Commission for Aeronautics, 1992.

32. F. B. Schneider. Enforceable security policies. Computer Science Technical Report TR98-1644, Cornell University, Computer Science Department, September 1998.

33. S. Schulz. System abstract: E 0.3. In H. Ganzinger (ed.), Proc. 16th Intl. Conf. Automated Deduction, Lect. Notes Artificial Intelligence 1421, pp. 297-301. Springer, 1999.

34. Z. Shao, C. League, and S. Monnier. Implementing typed intermediate language. In Proc. 1998 ACM SIGPLAN Intl. Conf. Functional Programming, pp. 313-323. 1998.

35. G. Stenz and A. Wolf. E-SETHEO: Design configuration and use of a parallel theorem prover. In N. Foo (ed.), Proc. of the 12th Australian Joint Conf. on Artificial Intelligence, Lect. Notes Artificial Intelligence 1747, pp. 231-243. Springer, 1999.

36. W. Visser, K. Havelund, G. Brat, and S. Park. Model checking programs. In P. Alexander and P. Flener (eds.), Proc. 15th Intl. Conf. Automated Software Engineering, pp. 3-12. IEEE Comp. Soc. Press, 2000.

37. W. Reif. The KIV Approach to Software Verification. In M. Broy and S. Jähnichen (eds.), KORSO: Methods, Languages and Tools for the Construction of Correct Software, Lect. Notes Comp. Sci. 1009, pp. 339-370. Springer, 1995.

38. C. Weidenbach, B. Gaede, and G. Rock. Spass and Flotter version 0.42. In M. A. McRobbie and J. K. Slaney (eds.), Proc. 13th Intl. Conf. Automated Deduction, Lect. Notes Artificial Intelligence 1104, pp. 141-145. Springer, 1996.

39. J. Whittle, J. Van Baalen, J. Schumann, P. Robinson, T. Pressburger, J. Penix, P. Oh, M. Lowry, and G. Brat. Amphion/NAV: Deductive synthesis of state estimation software. In Feather and Goedicke [10], pp. 395-399.

40. G. Winskel. The Formal Semantics of Programming Languages: An Introduction. The MIT Press, 1993.

41. N. Wirth. Programming in Modula-2. Springer, 4th edition, 1988.

42. H. Xi and F. Pfenning. Eliminating array bound checking through dependent types. In Proc. ACM Conf. on Programming Language Design and Implementation 1998, pp. 249-257. ACM Press, 1998. Published as SIGPLAN Notices 33(5). 